\title{
Dimensional oscillation
}

\author{
A fast variation of energy embedding gives good results with the \\ AMBER potential energy function
}

MARK E. SNOW* and GORDON M. CRIPPEN**

* Scientific Computation Group, and ${ }^{* *}$ College of Pharmacy, University of Michigan, Ann Arbor, MI, USA

Received 25 August 1990, accepted for publication 16 February 1991

\begin{abstract}
The structure of the AMBER potential energy surface of the cyclic tetrapeptide cyclotetrasarcosyl is analyzed as a function of the dimensionality of coordinate space. It is found that the number of local energy minima decreases as the dimensionality of the space increases until some limit at which point equipotential subspaces appear. The applicability of energy embedding methods to finding global energy minima in this type of energy-conformation space is explored. Dimensional oscillation, a computationally fast variant of energy embedding is introduced and found to sample conformation space widely and to do a good job of finding global and near-global energy minima.
\end{abstract}

Key words: energy embedding: molecular modeling: multiple-minimum problem; peptide and protein structure

Molecular mechanics methods have been an invaluable tool in the study of the molecular conformation of peptides and proteins. There are, however, two well-known limitations to studies based on molecular mechanics methods. One limitation is due to the extent to which the potential functions and their parameterizations do not reflect the true energy surface of the molecule. The other limitation arises from the very large number of local minima in the energy surface of even a small molecule.

In general, it is possible for a multiple minimum problem to be simply an artifact of the space in which the problem is posed. One could imagine three points, connected by springs such that the optimal separation between each pair of points is one unit. If these points are forced to reside on the line, all of the interactions cannot be optimally satisfied simultaneously. Relaxing the problem to two dimensions, however, allows all the interactions to be satisfied. In this case, the global minimum would be degenerate with respect to rotation, translation, and mirror inversion.

Energy embedding methods have been shown to be an effective method for finding the global minimum of such simple test functions (1). These methods locate the minimum in a higher dimensional space, then use this information to locate minima in the space in which the problem was initially posed. This can be done using principle component analysis, augmented Lagrangian (2) methods or a variety of other techniques. The embedding approach has been particularly useful with potentials that are a function only of the distances between pairs of atoms. Examples from chemistry include specifically designed folding potentials $(3,4)$.

Potentials depending only on distances between pairs of atoms may (depending on the details of the interatomic interaction terms) have the property that, in a high enough dimensional space, all of the distance constraints can be satisfied simultaneously resulting in a unique global energy minimum. Of course, this minimum only exists in the high dimensional space. The energy surface in 3-space retains its complexity and its multiple minima.

Commonly used molecular mechanics potentials like AMBER (5) which contain energy terms for bond angles, dihedral angles and so forth are not embeddable, that is there is no space in which all of the terms in the energy equation can be satisfied simultaneously. Even so, a preliminary study of energy embedding with the AMBER potential revealed that the global energy minimum of the dipeptide $N$-acetyl-L-alanine$N^{\prime}$-methylamide was found (6).

Preliminary minimization in higher-dimensional spaces followed by embedding to 3 -space have also 
been used in the determination of structures from NMR data ${ }^{\dagger}$ and with distance constraints derived from evolutionary data (7).

In this paper, we use the cyclic tetrapeptide cyclotetrasarcosyl as a model system to study the structure of the AMBER potential function in higher dimensional spaces, and its implications for the energy embedding problem.

\section{STRUCTURE OF THE ENERGY SURFACE AS A FUNCTION OF DIMENSIONALITY}

That a unique energy minimum was found in 11-space for $N$-acetyl-L-alanine- $N^{\prime}$-methylamide (6) is probably due to its small size and, as stated above, cannot be expected to be the case for molecular mechanicstype potentials in general. Intuitively, one would expect the number of local minima to drop as the dimension of the space increases until reaching some limit. At this point, further increasing the dimension of the space will create equipotential subspaces out of the local minima. To see why this is the case, imagine trying to embed a molecule in a space with many more dimensions that the molecule has degrees of freedom.

To explore this idea, we generated 60 random starting conformations for cyclotetrasarcosyl. AMBER energy minimizations were performed in 3-space, in 4-space, in 5-space, and so on from each of the 60 starting conformations. To analyze this data. distance matrices were constructed for each of the minimized structures. Pairwise analysis of all the distance matrices was used to analyze the conformations of the resultant structures and to locate groups of structures which had converged to the same local energy minimum. Note that such an analysis allows one to quickly identify structures in the same local energy minimum even though the structures in question may be embedded in spaces of different dimension. The details of this analysis follow.

\section{STRUCTURE NOMENCLATURE AND GENERATION OF STARTING CONFORMATIONS}

An all-trans extended tetrasarcosyl was built with AMBER geometry. Starting from this structure, backbone dihedral angles were rotated to produce extended structures with all-cis, cis-trans-cis and transcis-trans geometries. These structures were named $t 0$, $c 0, d 0$, and $u 0$, respectively. An additional all-cis structure with ring closure enforced was named $e 0$. For the other structures, ring closure was not enforced at this time, but was quickly achieved during energy minimization. Fifty-five additional structures were built in the following way: One of the starting structures $t 0$, $c 0, d 0$, or $u 0$, was selected at random. Three phi or psi

\footnotetext{
$\dagger$ This has been used, for example, in the groups of Havel, of Hare, and of Blaney.
}

angles in the structure were selected at random and each was rotated by a random amount between $-180^{\circ}$ and $+180^{\circ}$ (names and values for all angles follow IUPAC-IUB convention (8)). These structures were named with the appropriate letter $t, c, d$, or $u$ followed by an integer from 1 to 55 . Each of these 60 starting points was then AMBER minimized in 3space, in 4-space, and so on through 7-space, to produce a total of 300 minimized structures. The energy minimized structures are named with the starting structure name followed by $r 3$ for a 3-space minimized structure, $r 4$ for a 4 -space minimized structure and so on. Thus the 7-space minimized structure from the $u 51$ starting point is $u 51 r 7$.

\section{Distribution of local minima}

Since different starting geometries may converge to a common local minimum during energy minimization, the 60 minimized structures in a space of any given dimension will not necessarily have unique conformations. To look at the distribution of local minima in a given space, a distance matrix was constructed for each of the 60 minimized structures in that space. From these distance matrices was constructed a symmetric $60 \times 60$ matrix giving the root mean square distance deviation between each pair of structures. Such a matrix quickly reveals the number of local minima located and which structures have converged to which minima. These results are summarized in Table 1.

Fig. 1 is a plot of the number of local minima found, and their energies as a function of the dimension of the space. While we do not believe that we have rigorously located all local minima, the results make it clear that 1) for 3-space through 5-space, the number of local minima decreases as the dimension of the space increases, 2) in 6-space and beyond, one continues to observe the same local minima which were found in 5 -space, and 3) there is no space in which a unique energy minimum is observed.

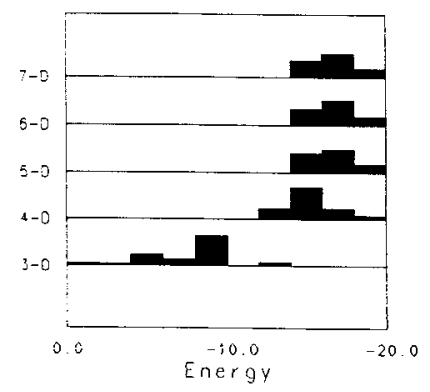

FIGURE !

Energy and density of local minima as a function of spatial dimension. For each spatial dimension, the number of occurrences is plotted as a histogram vs. the AMBER energy in $\mathrm{kcal} / \mathrm{mol}$. The histogram is divided into bins of width $2.0 \mathrm{kcal} / \mathrm{mol}$. 
Dimensional oscillation

TABLE 1

Energies and occurrences of local energy minima of cyclotetrasarcosyl in spaces of different dimension. See text for conformation naming conventions. When a higher-dimensional conformation is equivalent to a conformation in a lower-dimensional space, the name of the lowerdimensional conformation is given in parentheses

\begin{tabular}{|c|c|c|c|}
\hline $\begin{array}{l}\text { Dimension } \\
\text { of space }\end{array}$ & $\begin{array}{c}\text { Conformation } \\
\text { name }\end{array}$ & $\begin{array}{c}\text { Energy } \\
(\mathrm{kcal} / \mathrm{mol})\end{array}$ & Occurrences \\
\hline \multirow[t]{38}{*}{ 3-space } & $\mathrm{m} 0$ & -9.0 & $\begin{array}{l}\mathrm{c} 0 \mathrm{r} 3, \mathrm{c} 7 \mathrm{r} 3, \mathrm{c} 9 \mathrm{r} 3 \\
\mathrm{c} 29 \mathrm{r} 3, \mathrm{~d} 03\end{array}$ \\
\hline & $\mathrm{ml}$ & -9.8 & $\mathrm{c} 3 \mathrm{r} 3, \mathrm{c} 40 \mathrm{r} 3$ \\
\hline & $\mathrm{m} 2$ & -8.6 & c16r3 \\
\hline & $\mathrm{m} 3$ & -9.5 & $\mathrm{cl} 17 \mathrm{r} 3$ \\
\hline & $\mathrm{m} 4$ & -8.6 & $\mathrm{c} 27 \mathrm{r} 3$ \\
\hline & $\mathrm{m} 5$ & -0.8 & $\mathrm{c} 28 \mathrm{r} 3$ \\
\hline & $\mathrm{m} 6$ & -9.5 & $\mathrm{e} 30 \mathrm{r} 3, \mathrm{u} 43 \mathrm{r} 3$ \\
\hline & $\mathrm{m} 7$ & -4.1 & $\mathrm{c} 50 \mathrm{r} 3$ \\
\hline & $\mathrm{m} 8$ & -8.8 & $\begin{array}{l}\mathrm{d} 8 \mathrm{r} 3, \mathrm{~d} 19 \mathrm{r} 3, \mathrm{~d} 21 \mathrm{r} 3, \\
\mathrm{~d} 22 \mathrm{r} 3, \mathrm{~d} 25 \mathrm{r} 3, \mathrm{~d} 35 \mathrm{r} 3, \\
\mathrm{~d} 53 \mathrm{r} 3, \mathrm{~d} 55 \mathrm{r} 3\end{array}$ \\
\hline & $\mathrm{m} 9$ & -7.2 & $\mathrm{~d} 14 \mathrm{r} 3$ \\
\hline & $\mathrm{m} 10$ & -9.3 & $\mathrm{~d} 23 \mathrm{r} 3$ \\
\hline & $\mathrm{m} 11$ & -6.7 & $\mathrm{~d} 26 \mathrm{r} 3$ \\
\hline & $\mathrm{m} 12$ & -8.7 & $\mathrm{~d} 37 \mathrm{r} 3$ \\
\hline & $\mathrm{m} 13$ & -6.3 & $\mathrm{~d} 38 \mathrm{r} 3$ \\
\hline & $\mathrm{m} 14$ & -7.1 & $\mathrm{~d} 4 \operatorname{lr} 3$ \\
\hline & $\mathrm{m} 15$ & -0.9 & $d 45 r 3$ \\
\hline & $\mathrm{m} 16$ & -6.1 & $\mathrm{e} 0 \mathrm{r} 3$ \\
\hline & $\mathrm{ml} 7$ & -12.7 & $\mathrm{t} 0 \mathrm{r} 3, \mathrm{t} 42 \mathrm{r} 3, \mathrm{t} 46 \mathrm{r} 3$ \\
\hline & $\mathrm{m} 18$ & -8.3 & $\operatorname{tln} 3$ \\
\hline & $\mathrm{m} 19$ & -6.3 & $\mathrm{t} 2 \mathrm{r}^{3}$ \\
\hline & $\mathrm{m} 20$ & -1.9 & $\mathrm{t} 5 \mathrm{r} 3$ \\
\hline & $\mathrm{m} 21$ & -5.0 & $\begin{array}{l}\mathrm{t} 10 \mathrm{r} 3, \mathrm{t} 13 \mathrm{r} 3, \mathrm{t} 34 \mathrm{r} 3 \\
\mathrm{t} 36 \mathrm{r} 3, \mathrm{t} 1 \mathrm{r} 3\end{array}$ \\
\hline & $\mathrm{m} 22$ & -2.6 & $t 44 r^{3}$ \\
\hline & $\mathrm{m} 23$ & -8.0 & $t 49 r 3$ \\
\hline & $\mathrm{m} 24$ & -8.3 & $\mathrm{t} 54 \mathrm{r} 3$ \\
\hline & $\mathrm{m} 25$ & -6.8 & u0r3 \\
\hline & $\mathrm{m} 26$ & -5.4 & $\mathrm{u} 4 \mathrm{r} 3, \mathrm{u} 6 \mathrm{r} 3$ \\
\hline & $\mathrm{m} 27$ & -8.0 & ullr3 \\
\hline & $\mathrm{m} 28$ & -12.3 & $\mathrm{ul} 2 \mathrm{r} 3$ \\
\hline & $\mathrm{m} 29$ & -8.7 & ul $15 \times 3, u 33 r 3$ \\
\hline & $\mathrm{m} 30$ & -9.3 & u $18 \mathrm{r} 3$ \\
\hline & $\mathrm{m} 31$ & -4.5 & $\mathrm{u} 20 \mathrm{r} 3$ \\
\hline & $\mathrm{m} 32$ & -5.6 & $\mathrm{u} 24 \mathrm{r} 3$ \\
\hline & $\mathrm{m} 33$ & -2.8 & $\mathrm{u} 31 \mathrm{r} 3$ \\
\hline & $\mathrm{m} 34$ & -5.7 & $\mathrm{u} 32 \mathrm{r} 2$ \\
\hline & $\mathrm{m} 35$ & -8.7 & u39r3 \\
\hline & $\mathrm{m} 36$ & -8.6 & $\mathrm{u} 47 \mathrm{r} 3, \mathrm{u} 48 \mathrm{r} 3$ \\
\hline & $\mathrm{m} 37$ & -4.5 & $\mathrm{u} 52 \mathrm{r} 3$ \\
\hline \multirow[t]{8}{*}{ 4-space } & $\mathrm{m} 38$ & -13.4 & $\mathrm{cor} 4$ \\
\hline & m39 & -17.9 & $\begin{array}{l}\mathrm{c} 3 \mathrm{r} 4, \mathrm{c} 4 \mathrm{r} 4, \mathrm{u} 20 \mathrm{r} 4 \\
\mathrm{u} 47 \mathrm{r} 4\end{array}$ \\
\hline & $\mathrm{m} 40$ & -18.0 & $\begin{array}{l}\mathrm{c} 7 \mathrm{r} 4, \mathrm{c} 50 \mathrm{r} 4, \mathrm{e} 0 \mathrm{r} 4 \\
\mathrm{u} 43 \mathrm{r} 4\end{array}$ \\
\hline & $\mathrm{m} 41$ & -17.2 & $\mathrm{c} 9 \mathrm{r} 4$ \\
\hline & $\mathrm{m} 42$ & -17.9 & $\mathrm{cl} 6 \mathrm{r} 4$ \\
\hline & $\mathrm{m} 43$ & -13.5 & $\mathrm{cl} 7 \mathrm{r} 4, \mathrm{c} 27 \mathrm{r} 4$ \\
\hline & $\mathrm{m} 44$ & -15.1 & $\mathrm{c} 28 \mathrm{r} 4$ \\
\hline & $\mathrm{m} 45$ & -16.1 & $\mathrm{c} 29 \mathrm{r} 4$ \\
\hline
\end{tabular}

TABLE I continued

\begin{tabular}{|c|c|c|c|}
\hline $\begin{array}{l}\text { Dimension } \\
\text { of space }\end{array}$ & $\begin{array}{c}\text { Conformation } \\
\text { name }\end{array}$ & $\begin{array}{c}\text { Energy } \\
(\mathrm{kcal} / \mathrm{mol})\end{array}$ & Occurrences \\
\hline & $\mathrm{m} 46$ & -13.8 & $\mathrm{c} 30 \mathrm{r} 4$ \\
\hline & $\mathrm{m} 47$ & -15.7 & $\begin{array}{l}\mathrm{d} 0 \mathrm{r} 4, \mathrm{~d} 19 \mathrm{r} 4, \mathrm{~d} 2 \mathrm{lr} 4, \\
\mathrm{~d} 25 \mathrm{r} 4, \mathrm{~d} 26 \mathrm{r} 4, \mathrm{~d} 53 \mathrm{r} 4 \\
\mathrm{t} 2 \mathrm{r} 4\end{array}$ \\
\hline & $\mathrm{m} 48$ & -12.5 & $\mathrm{~d} 8 \mathrm{r} 4$ \\
\hline & $\mathrm{m} 49$ & -17.5 & $\mathrm{dI} 4 \mathrm{r} 4$ \\
\hline & $\mathrm{m} 50$ & -14.9 & $\mathrm{~d} 22 \mathrm{r} 4, \mathrm{~d} 37 \mathrm{r} 4, \mathrm{~d} 38 \mathrm{r} 4$ \\
\hline & $\mathrm{m} 51$ & -16.7 & $\mathrm{~d} 35 \mathrm{r} 4$ \\
\hline & $\mathrm{m} 52$ & -14.3 & $\mathrm{~d} 41 \mathrm{r} 4, \mathrm{t} 42 \mathrm{r} 4$ \\
\hline & $\mathrm{m} 53$ & -14.8 & $\mathrm{~d} 45 \mathrm{r} 4, \mathrm{~d} 55 \mathrm{r} 4, \mathrm{t} 3 \mathrm{r} 4$ \\
\hline & $\mathrm{m} 54$ & -15.8 & tor $4, t 46 r 4, t 54 r 4$ \\
\hline & m55 & -13.4 & $\mathrm{t} 1 \mathrm{r} 4, \mathrm{t} 13 \mathrm{r} 4$ \\
\hline & $\mathrm{m} 56$ & -12.4 & $t 5 r 4$ \\
\hline & $\mathrm{m} 57$ & -14.2 & t10r4 \\
\hline & $\mathrm{m} 58$ & -14.6 & $\mathrm{t} 36 \mathrm{r} 4, \mathrm{t} 51 \mathrm{r} 4$ \\
\hline & $\mathrm{m} 59$ & -14.7 & $\mathrm{t} 44 \mathrm{r} 4$ \\
\hline & $\mathrm{m} 60$ & -14.5 & $\mathrm{t} 49 \mathrm{r} 4$ \\
\hline & $\mathrm{m} 61$ & -13.5 & uor 4 \\
\hline & $\mathrm{m} 62$ & -14.8 & $\begin{array}{l}\text { u4r4, u6r4, u24r4. } \\
\text { u33r4 }\end{array}$ \\
\hline & $\mathrm{m} 63$ & -15.3 & ullr4 \\
\hline & $\mathrm{m} 64$ & -17.5 & $\mathrm{u} 12 \mathrm{r} 4$ \\
\hline & $\mathrm{m} 65$ & -15.7 & u15r4 \\
\hline & m66 & -12.9 & $\mathrm{u} 18 \mathrm{r} 4, \mathrm{u} 31 \mathrm{r} 4$ \\
\hline & $\mathrm{m} 67$ & -16.8 & $\mathrm{u} 32 \mathrm{r} 4$ \\
\hline & $\mathrm{m} 68$ & -14.7 & $\mathrm{u} 39 \mathrm{r} 4$ \\
\hline & m69 & -15.2 & $\mathrm{u} 48 \mathrm{r} 4, \mathrm{u} 52 \mathrm{r} 4$ \\
\hline \multirow[t]{17}{*}{ 5-space } & $\mathrm{m} 70$ & -18.2 & $\begin{array}{l}\text { c0r5, c3r5, c16r5, } \\
\text { c17r5, c27r5, c30rs, } \\
\text { c40r5, u0r5, u43r5, } \\
\text { e0r5, u20r5, u47r5 }\end{array}$ \\
\hline & $\mathrm{m} 71$ & -17.6 & $\begin{array}{l}\mathrm{c} 7 \mathrm{r} 5, \mathrm{c} 9 \mathrm{r} 5, \mathrm{c} 28 \mathrm{r} 5 \\
\mathrm{c} 29 \mathrm{r} 5, \mathrm{c} 50 \mathrm{r} 5\end{array}$ \\
\hline & $\mathrm{m} 72$ & -14.6 & $\mathrm{~d} 0 \mathrm{r} 5$ \\
\hline & $\mathrm{m} 73$ & -17.0 & $\begin{array}{l}\mathrm{d} 8 \mathrm{r} 5, \mathrm{~d} 19 \mathrm{r} 5, \mathrm{~d} 21 \mathrm{r} 5, \\
\mathrm{~d} 26 \mathrm{r} 5, \mathrm{~d} 35 \mathrm{r} 5, \mathrm{~d} 45 \mathrm{r} 5, \\
\mathrm{~d} 53 \mathrm{r} 5, \mathrm{t} 1 \mathrm{r} 5, \mathrm{t} 2 \mathrm{r} 5, \\
\mathrm{t} 5 \mathrm{r} 5, \mathrm{t} 34 \mathrm{r} 5\end{array}$ \\
\hline & $\mathrm{m} 74$ & -17.7 & $\begin{array}{l}\mathrm{d} 14 \mathrm{r} 5, \mathrm{~d} 22 \mathrm{r} 5, \mathrm{~d} 25 \mathrm{r} 5 \\
\mathrm{~d} 37 \mathrm{r} 5, \mathrm{~d} 28 \mathrm{r} 5, \mathrm{~d} 55 \mathrm{r} 5\end{array}$ \\
\hline & $\mathrm{m} 75$ & -15.8 & $\mathrm{~d} 23 \mathrm{r} 5$ \\
\hline & $\mathrm{m} 76$ & -14.4 & $\mathrm{~d} 41 \mathrm{r} 5, \mathrm{t} 36 \mathrm{r} 5$ \\
\hline & $\mathrm{m} 77$ & -15.8 & $\begin{array}{l}\text { t0r } 5, \mathrm{t} 42 \mathrm{r} 5, \mathrm{t} 46 \mathrm{r} 5 \\
\mathrm{t} 54 \mathrm{r} 5\end{array}$ \\
\hline & $\mathrm{m} 78$ & -14.7 & t $10 \mathrm{r} 5$ \\
\hline & $\mathrm{m} 79$ & -14.8 & $\mathrm{t} 13 \mathrm{r} 5, \mathrm{t} 51 \mathrm{r} 5$ \\
\hline & $\mathrm{m} 80$ & -14.5 & $\mathrm{t} 44 \mathrm{r} 5, \mathrm{t} 49 \mathrm{r} 5$ \\
\hline & $\mathrm{m} 81$ & -15.3 & $\begin{array}{l}\mathrm{u} 4 \mathrm{r} 5, \mathrm{u} 6 \mathrm{r} 5, \mathrm{u} 24 \mathrm{r} 5 \\
\mathrm{u} 33 \mathrm{r} 5, \mathrm{u} 48 \mathrm{r} 5, \mathrm{u} 52 \mathrm{r} 5\end{array}$ \\
\hline & $\mathrm{m} 82$ & -16.0 & ul1rs \\
\hline & $\mathrm{m} 83$ & -17.7 & ul2r 5 \\
\hline & $\mathrm{m} 84$ & -17.1 & ul 5 r 5 \\
\hline & $\mathrm{m} 85$ & -15.2 & u18r5, u31r5 \\
\hline & $\mathrm{m} 86$ & -17.0 & $\mathrm{u} 32 \mathrm{r} 5, \mathrm{u} 39 \mathrm{r} 5$ \\
\hline 6-space & $\mathrm{m} 87(\mathrm{~m} 70)$ & -18.1 & $\begin{array}{l}\text { c0r6, c3r6, c16r6, } \\
\text { c17r6, c27r6, c30r6, } \\
\text { c40r6, e0r6, u0r6, }\end{array}$ \\
\hline
\end{tabular}


TABLE I continued

\begin{tabular}{|c|c|c|c|}
\hline \multirow[t]{16}{*}{$\begin{array}{l}\text { Dimension } \\
\text { of space }\end{array}$} & $\begin{array}{c}\text { Conformation } \\
\text { name }\end{array}$ & $\begin{array}{c}\text { Energy } \\
(\mathrm{kcal} / \mathrm{mol})\end{array}$ & Occurrences \\
\hline & & & $\begin{array}{l}u 20 r 6, u 39 r 6, u 43 r 6 \text {, } \\
u 47 r 6\end{array}$ \\
\hline & $\mathrm{m} 88(\mathrm{~m} 7 \mathrm{l})$ & -17.6 & $\begin{array}{l}c 7 r 6, c 9 r 6, c 28 r 6 \\
\text { c29r6. c50r6, u18r6 }\end{array}$ \\
\hline & $\mathrm{m} 89(\mathrm{~m} 73)$ & -16.9 & $\begin{array}{l}\text { d0r6, d8r6, d19r6, } \\
\text { d21r6, d23r6, d35r6. } \\
\text { d45r6. d53r6, d55r6, } \\
\text { 11r6. t2r6, t5r6, t34r6 }\end{array}$ \\
\hline & $\mathrm{m} 90(\mathrm{~m} 74)$ & -17.7 & $\begin{array}{l}\text { d14r6. d22r6. d25r6. } \\
\text { d26r6. d37r6. d38r6 }\end{array}$ \\
\hline & $\mathrm{m} 91(\mathrm{~m} 76)$ & -14.3 & $\mathrm{~d} 41 \mathrm{r6}, \mathrm{t} 36 \mathrm{r} 6 . \mathrm{t} 51 \mathrm{r} 6$ \\
\hline & $\mathrm{m} 92(\mathrm{~m} 77)$ & -15.7 & 10r6. $: 46 \mathrm{r} 6$ \\
\hline & $\mathrm{m} 93(\mathrm{~m} 78)$ & -14.7 & $110 r 6.142 r 6 .+54 r 6$ \\
\hline & $\mathrm{m} 94(\mathrm{~m} 79)$ & -14.8 & $113 \pi 6$ \\
\hline & $\mathrm{m} 93(\mathrm{~m} 80)$ & -14.5 & $144 \mathrm{r} 6,149 \mathrm{r} 6$ \\
\hline & $\mathrm{m} 96(\mathrm{~m} 8 \mathrm{I})$ & -15.3 & $\begin{array}{l}\text { u4r6. u6r6, u24r6, } \\
\text { u48r6. u52r6 }\end{array}$ \\
\hline & $\mathrm{m} 97(\mathrm{~m} 82)$ & -16.0 & ullir 6 \\
\hline & $\mathrm{m} 98(\mathrm{~m} 83)$ & -17.7 & u12r6 \\
\hline & $\mathrm{m} 99(\mathrm{~m} 84)$ & -17.1 & u 15 r6. u33r6 \\
\hline & $\mathrm{m} 100(\mathrm{~m} 85)$ & -15.2 & u31r6 \\
\hline & $\mathrm{m} 101(\mathrm{~m} 86)$ & -17.0 & u32r6 \\
\hline \multirow[t]{16}{*}{ 7-space } & $\mathrm{m} 102(\mathrm{~m} 70)$ & -18.1 & $\begin{array}{l}\text { c0r7, c3r7, c16r7. } \\
\text { c17r7. c27r7. c30r7 } \\
\text { c40r7. e0r7. u0r7. } \\
\text { u20r7, u39r7. u43r7. } \\
\text { u47r } 7\end{array}$ \\
\hline & $\mathrm{m} 103(\mathrm{~m} 71)$ & -17.5 & $\begin{array}{l}\text { c7r7. c9r7. c28r7. } \\
\text { c29r7. c50r7. c31r7 }\end{array}$ \\
\hline & $\mathrm{m} 104(\mathrm{~m} 73)$ & -17.0 & $\begin{array}{l}\text { d0r7, d8r7, d19r7. } \\
\text { d21r7, d23r7, d25r7, } \\
\text { d35r7, d45r7, d53r7. } \\
\text { d55r7, t1r7, t2r7. } \\
134 r 7\end{array}$ \\
\hline & $\mathrm{m} 105(\mathrm{~m} 74)$ & -17.8 & $\begin{array}{l}\text { d14r7. d22r7, d26r7, } \\
\text { d37r7. d } 38 \text { r } 7\end{array}$ \\
\hline & $\mathrm{m} 106(\mathrm{~m} 76)$ & -14.3 & $\mathrm{~d} 41 \mathrm{r} 7, \mathrm{t} 36 \mathrm{r} 7, \mathrm{t51r7}$ \\
\hline & $\mathrm{ml07}(\mathrm{m} 77)$ & -15.7 & $\begin{array}{l}t 0 r 7, t 42 r 7, t 46 r 7 \\
154 r 7\end{array}$ \\
\hline & $\mathrm{ml} 108(\mathrm{~m} 80)$ & -14.4 & $15 r 7.149 r 7$ \\
\hline & $\mathrm{ml} 109(\mathrm{~m} 78)$ & -14.7 & 11057 \\
\hline & $\mathrm{m} 110(\mathrm{~m} 79)$ & -14.8 & $113 r 7$ \\
\hline & $\mathrm{m} 111$ & -15.0 & $144 \mathrm{r} 7$ \\
\hline & $\mathrm{m} 112(\mathrm{~m} 81)$ & -15.3 & $\begin{array}{l}\text { u } 4 r 7, \text { u } 6 r 7, u 24 r 7 \\
\text { u } 48 \text { r } 7, \text { u } 52 r 7\end{array}$ \\
\hline & $\mathrm{m} 113(\mathrm{~m} 82)$ & -16.0 & u 1157 \\
\hline & $\mathrm{m} 114(\mathrm{~m} 83)$ & -17.7 & $\mathrm{u} 12 \mathrm{r} 7$ \\
\hline & $\mathrm{ml15}$ & -17.1 & u15r7, u33r7 \\
\hline & $\mathrm{mll} 16(\mathrm{~m} 85)$ & -15.2 & $\mathrm{u} 18 \mathrm{r} 7$ \\
\hline & $\mathrm{mll} 7(\mathrm{~m} 86)$ & -17.0 & $\mathrm{u} 32 \mathrm{r} 7$ \\
\hline
\end{tabular}

\section{ENERGY EMBEDDING}

Energy embedding (1) has been found to be an effective technique for finding global 3-space energy minima for potentials which have a unique global minimum in a higher dimensional space. The fact that molecular mechanics-type potentials do not have a unique global minimum in any space opens the question of whether energy embedding will be an effective technique for this type of potential, and of which high-dimensional conformation should be used as a starting point for embedding to 3 -space. The high computational cost of the embedding procedure combined with the large number of high-dimensional local minima preclude the possibility of embedding from all high-dimensional energy minima.

Energy embedding was attempted from the lowest energy structure in 6-space (conformation $\mathrm{m} 87$, 6space energy $=-18.12 \mathrm{kcal} / \mathrm{mol})$. The embedding procedure consisted of several iterations of minimizing the augmented Lagrangian (2) function

$$
\begin{aligned}
L(\vec{x}, \vec{i}, \vec{w}, W)= & E(\vec{x})+\vec{\lambda} \cdot \vec{g}+\left(\vec{w}^{T}\right) \cdot \vec{g} \\
& +\frac{1}{2} W(\vec{g} \cdot \vec{g})
\end{aligned}
$$

where $\vec{x}$ is the vector of spatial coordinates of the atoms, $E$ is the conventional AMBER energy of conformation $\vec{x}, \vec{\lambda}$ is a vector of Lagrange multipliers, $\vec{g}$ is a vector of coordinates to be eliminated, i.e. in this case the 4th through 6th coordinate of each atom, $W$ is a weight on the penalty term, and $\vec{w}$ is a vector of separate weights on the individual unwanted coordinates.

Initially, in iteration, $0, \vec{\lambda}=0$ while $W$ and $\vec{w}$ are small, usually of the order of 0.01. An iteration consists of minimizing $L$, then updating the weights and Lagrange multipliers according to

$$
\begin{aligned}
& \lambda_{i}^{(k+1)}=\lambda_{i}^{(k)}+W^{(k)} g_{i}^{(k+1)} \\
& W^{(k+1)}= \begin{cases}a W^{(k)} & \text { if }\left\|g^{(k+1)}\right\|>b\left\|g^{(k)}\right\| \\
W^{(k)} & \text { otherwise }\end{cases} \\
& w_{i}^{(k+1)}= \begin{cases}\mathrm{cw}_{i}^{(k)} & \text { if } g_{i}^{2}>d \frac{\left\|g^{(k+1)}\right\|^{2}}{N} \\
w_{i}^{(k)} \text { otherwise } & \end{cases}
\end{aligned}
$$

where $a, b, c$, and $d$ are constants such that $1<a, c$, $d$ and $0<b \leqslant 1$. In order for the embedding to proceed slowly enough, $a$ is ideally about 1.2 and no greater than 1.5. If $a$ is too large, the path becomes sensitive to small changes in parameters (9). Likewise $c$ should be no larger than 2.0 and is ideally about 1.5 . We have found 0.25 to be a good value for $b$ and 1.5 for $d$. The iterations continue until $\vec{g} \cdot \vec{g}$ is arbitrarily small $(<0.001)$.

Embedding from the $\mathrm{m} 87$ starting point resulted in a 3-space structure which lies in the previously observed conformation $\mathrm{m} 30$, and has energy $-9.32 \mathrm{kcal} /$ mol. While quite a good conformation, this is not the lowest energy conformation observed in 3-space. Note that this is a different (lower energy) conformation 
than the same starting structure refined to when simply minimized in 3-space.

The next attempt at energy embedding used the 6 -space structure $t 0 r 6(\mathrm{~m} 92)$ as a starting point. This starting point was chosen because the $t 0$ conformation refined to the lowest energy 3-space conformation using simple energy minimization. The embedding of this structure from 6-space did result in the 17 conformation (energy $=-12.67 \mathrm{kcal} / \mathrm{mol}$ ), the lowest energy 3-space conformation observed.

While it is encouraging that the lowest-energy 3space conformation was obtained in only two embedding attempts from 6-space, we remain somewhat cautious about the use of energy embedding as a general method for finding global 3-space minima for large molecules. As we have seen here, it cannot be assumed that the lowest energy conformation in the high-dimension spaces will necessarily embed to the lowest energy conformation in 3-space.

\section{DIMENSIONAL OSCILLATION}

Given the large computational expense of rigorously embedding from 6-space, and the fact that this procedure cannot guarantee finding a global energy minimum, we were led to wonder if a quick embedding procedure could be developed which would provide comparable benefit (sample conformation-space widely, find global or near-global minima) at considerably reduced expense. We define dimensional oscillation to be the following simple process: Start from some existing conformation in 3-space. Minimize to achieve the nearest local energy minimum in 4-space. This minimization need not be a rigorous second derivative minimization to locate the local minimum to high precision. From this 4-space structure, embed back to three dimensions. Again this can be done with fairly liberal initial values for the embedding weights and update schedules.

To test this procedure, we selected nine local minima in 3-space as starting points. From our sample of 60 minimized structures in 3-space, we selected the three best (lowest energy) structures, the three worst (highest energy) structures, and three other structures selected at random. Each of these nine starting points was submitted to one round of dimensional oscillation, using the embedding protocol with $W$ and all the $w_{i}$ initially set to $0.5, a, c=2.0, b=0.25$, and $d=1.5$. For six of the nine test cases, the dimensional oscillation procedure resulted in the structure moving to a new conformation of lower energy than the minimum in which it started. In one case, the structure returned to the same local minimum, and in two cases to a higher energy local minimum. It was also observed that two structures from different starting points moved to a common conformation during the procedure. These results are summarized in Table 2.
TABLE 2

Energies of nine cyclotetrasarcosyl structures before and after dimensional oscillation. Structures marked * underwent cis-trans isomerization during the procedure

\begin{tabular}{lcc}
\hline Conformation & $\begin{array}{c}\text { Energy before } \\
\text { oscillation }(\mathrm{kcal} / \mathrm{mol})\end{array}$ & $\begin{array}{c}\text { Energy after } \\
\text { oscillation (kcal/mol) }\end{array}$ \\
\hline $\mathrm{m} 17$ & -12.67 & -12.67 \\
$\mathrm{~m} 28$ & -12.18 & -4.39 \\
$\mathrm{~m} 1$ & -9.78 & -9.59 \\
\hline $\mathrm{m} 5^{*}$ & -0.80 & -4.87 \\
$\mathrm{~m} 15$ & -0.91 & -4.89 \\
$\mathrm{~m} 20$ & -1.92 & -2.88 \\
\hline $\mathrm{m} 0$ & -9.01 & -9.59 \\
$\mathrm{~m} 33^{*}$ & -2.82 & -4.89 \\
$\mathrm{~m} 37^{*}$ & -4.50 & -4.97 \\
\hline
\end{tabular}

Considering that all nine starting conformations were well minimized structures, and that three of these were the three lowest energy conformations, these results are encouraging. Note also that the lowest energy structure returned to the same conformation during the procedure.

In addition to finding good local energy minima, it also appears that dimensional oscillation samples conformation space very widely. This can be observed in plots of the cyclotetrasarcosyl dihedral angles before and after dimensional oscillation (Fig. 2), and is particularly apparent when considering that three of the nine structures subjected to dimensional oscillation underwent cis-trans isomerizations. These were conformations $\mathrm{m} 5, \mathrm{~m} 33$, and $\mathrm{m} 37$ (Table 2). Fig. 3 shows the conformation of $\mathrm{m} 37$ before and after oscillation.

\section{CONCERNING THE CYCLOTETRASARCOSYL STRUCTURE}

Of all the 3-space conformations we have observed for cyclotetrasarcosyl, two conformations, $\mathrm{m} 17$ and $\mathrm{m} 28$, have significantly lower energy than any of the others.

The structure of cyclotetrasarcosyl has been determined crystallographically (10), and the conformation observed in the crystal is the cis-trans-cis-trans conformation $\mathrm{m} 28$. Note that this conformation has the second lowest observed AMBER energy. The fact that the all-trans conformation $\mathrm{m} 17$ has slightly lower energy rates the question of whether this is indeed a lower energy conformation or whether it is an artifact of the AMBER potential and the parameterization used. Since the molecule has no groups capable of forming salt bridges or hydrogen bonds, and there are no noteworthy interactions between monomers in the crystal, it is unlikely that crystal packing forces contribute significantly to the stability of the conformation 

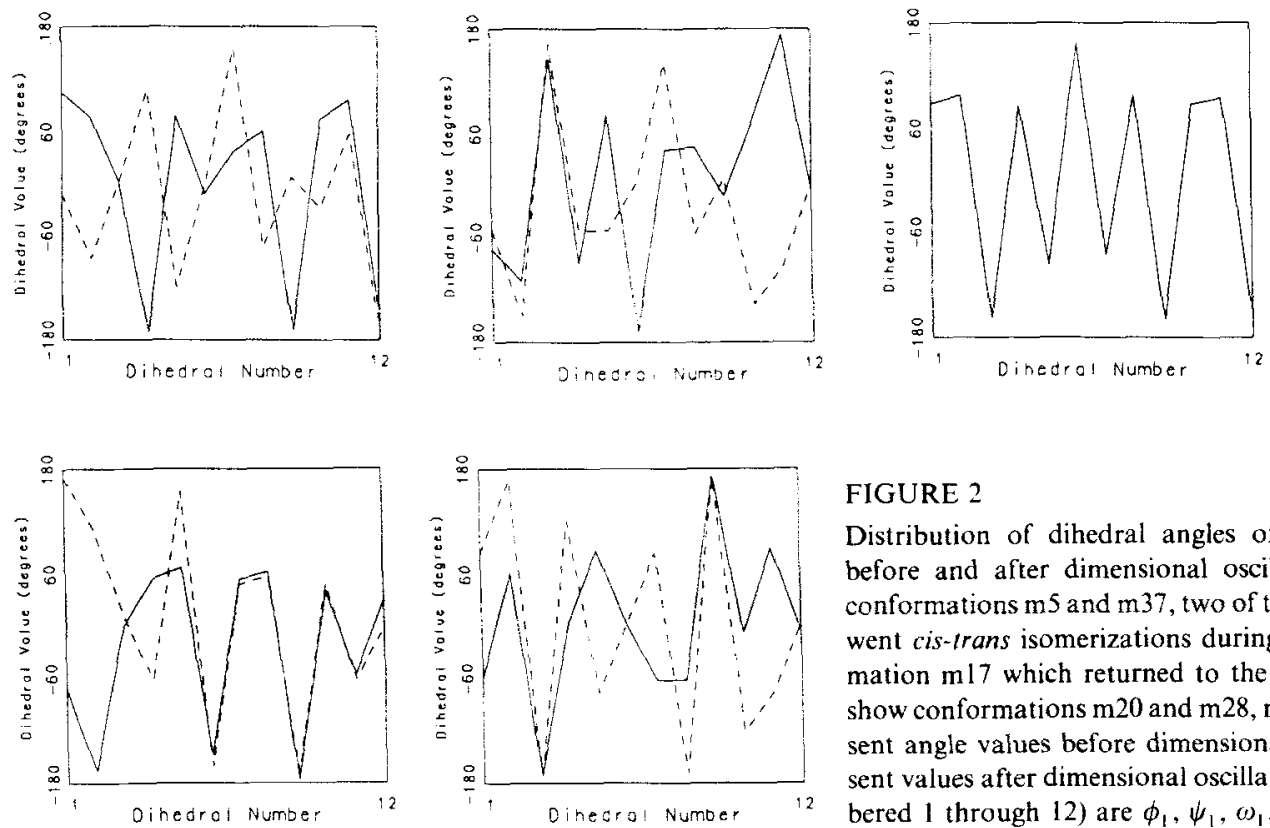

\section{FIGURE 2}

Distribution of dihedral angies of cyclotetrasarcosyl structures before and after dimensional oscillation. Fig. $2 a$ and $2 b$ show conformations $\mathrm{m} 5$ and $\mathrm{m} 37$, two of the conformations which underwent cis-trans isomerizations during oscillation. Fig. $2 \mathrm{c}$ is conformation $\mathrm{ml} 7$ which returned to the starting point. Fig. $2 \mathrm{~d}$ and $2 \mathrm{e}$ show conformations $m 20$ and $m 28$, respectively. Dashed lines represent angle values before dimensional oscillation, solid lines represent values after dimensional oscillation. The dihedral angles (numbered 1 through 12$)$ are $\phi_{1}, \psi_{1}, \omega_{1}, \phi_{2}, \ldots, \omega_{4}$.

observed in the crystal. Further, the available NMR data (11) are consistent with the cis-trans-cis-trans conformation being the only detectable conformer in solution. It thus seems likely that the lower energy calculated for the $\mathrm{m} 17$ conformation does not reflect the reality of the situation. It is possible that this is due to our choice of parameterization for the AMBER

potential. We used a distance dependent dielectric constant with a cutoff of 9.0 Angstroms, and a scale factor of 2.0 for 1-4 non-bonded and electrostatic interactions. The standard AMBER database does not contain a charge model for sarcosine or a model for all of the angles in that residue. Working from analogy with existing terms in the database, we added
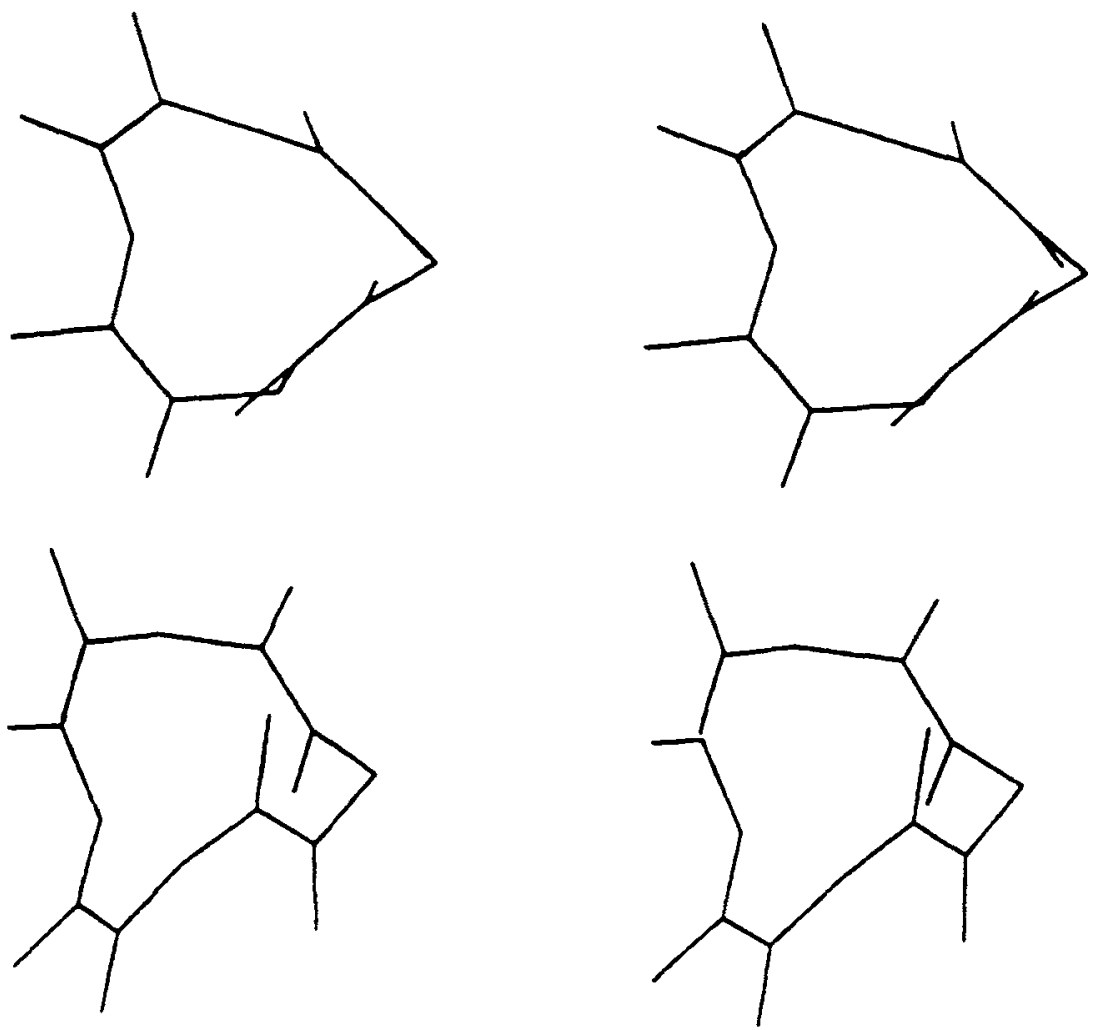

\section{FIGURE 3}

Stereo pair showing the conformation of the cyclotetrasarcosyl m37 structure before (top) and after dimensional oscillation. Note that the structure undergoes a cis-trans isomerization. 

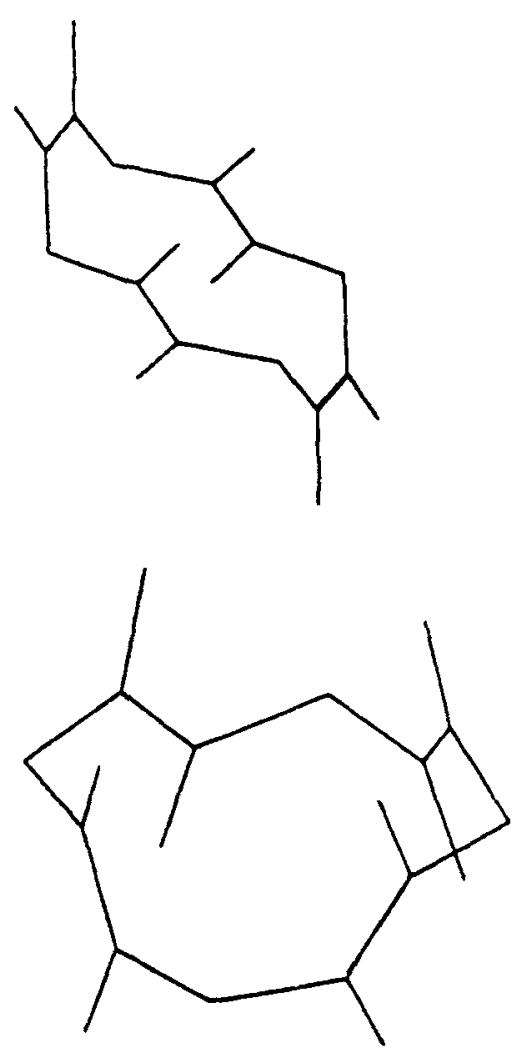
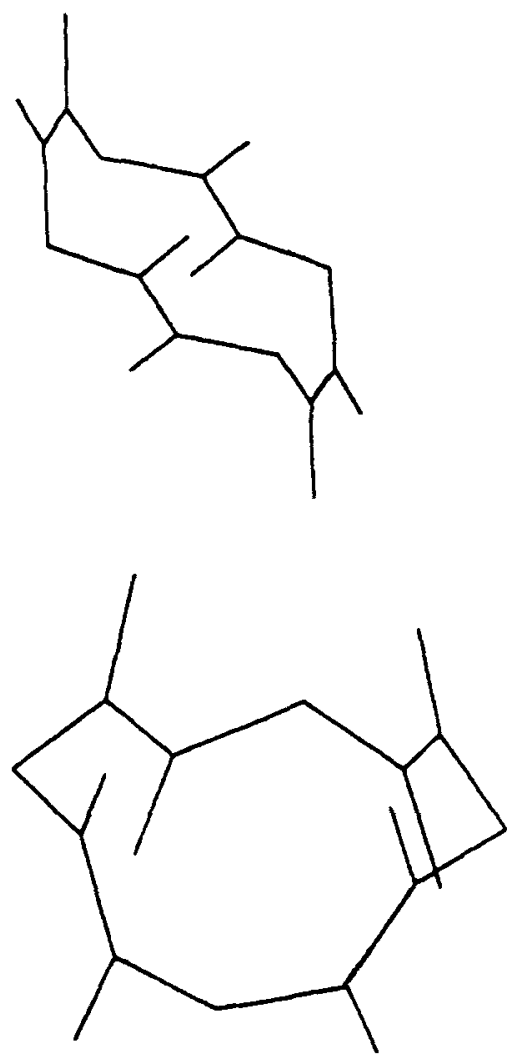

\section{FIGURE 4}

Stereo pair showing the $\mathrm{m} 28$ (top) and $\mathrm{ml} 7 \mathrm{conformations}$ of cyclotetrasarcosyl. These two conformations had AMBER energies of -12.27 and $-12.67 \mathrm{kcal} / \mathrm{mol}$, respectively, much lower than other conformations. The $\mathrm{m} 28$ conformation corresponds to that observed in the crystal structure and is consistent with the NMR data, although it has a slightly higher energy using this parameterization of the AMBER potential energy equation. a term for the C3-N-C2 angle with an optimal value of $116^{\circ}$ and a force constant of 50 . We used a charge model for sarcosine of $(\mathrm{N}=-0.52)$, $(\mathrm{CH} 3=+0.248),(\mathrm{C}=+0.526),(\mathrm{O}=-0.5)$. At the end of the study, we did do a test where we minimized from the $\mathrm{m} 17$ and $\mathrm{m} 28$ structures using a fixed dielectric constant. This did not change the order of the energies. Fig. 4 shows the structures of the $\mathrm{m} 17$ and $\mathrm{m} 28$ conformations.

\section{DISCUSSION}

In this paper we have examined how the AMBER conformational energy surface of cyclotetrasarcosyl varies as a function of the dimensionality of the space in which the molecule exists. In particular, we have found that the number of local energy minima decreases as the dimensionality of the space is increased until some limit at which point equipotential subspaces appear when the spatial dimension is further increased.

Energy embedding from the lowest energy conformation in 6-space did not achieve the 3-space global energy minimum, but embedding from a second 6space conformation did. This shows that energy embedding may prove to be a valuable tool for finding 3-space global minima, but that it cannot be assumed that embedding from the lowest minimum in a higher space will necessarily achieve the global 3-space minimum.

Dimensional oscillation appears to be a promising low-cost alternative to energy embedding for locating global and near-global energy minima. The method appears to sample conformation space widely and to consistently locate very good minima. We are currently evaluating the feasibility of applying the dimensional oscillation method to the study of larger molecules, and are hopeful that it will offer a promising alternative to existing molecular dynamics [12], monte carlo(13), sophisticated conformational search (14-19) and other methods of looking at peptides and proteins.

\section{REFERENCES}

1. Crippen, G. (1984) J. Comput. Chem. 5, 548

2. Bertsekas, D.P. (1982) Constrained Optimization and Lagrange Multiplier Methods. Academic Press, New York

3. Crippen, G.M. \& Ponnuswamy (1987) J. Comput. Chem. 8, 972.

4. Crippen, G.M. \& Snow, M.E. (1990) Biopolymers 29, 1479

5. Weiner, S.J., Kollman, P.A., Nguyen, D.T. \& Case, D.A. (1986) J. Comput. Chem. 7, 230

6. Crippen, G.M. (1987) J. Phys. Chem. 91, 6341

7. Havel, T.F. \& Snow, M.E. (1991) J. Mol. Biol. 217, 1

8. IUPAC-IUB Commission on Biochemical Nomenclature (1970) Biochemistry 9, 237 I

9. Snow, M.E. \& Crippen, G.M., unpublished results. 


\section{M.E. Snow and G.M. Crippen}

10. Groth, P. (1970) Acta Chem. Scand. 24, 780

11. Dale, J., Groth, P. \& Titlestad, K. (1977) Acta Chem. Scand. B31, 523

12. McCammon, J.A. \& Harvey, S.C. (1987) Dynamics of Proteins and Nucleic Acids. Cambridge University Press, and references therein.

13. See, for example, Le, Z. \& Scheraga, H.A. (1987) Proc. Natl. Acad. Sci. US 84, 6611

14. Shih, H.H-L., Brady, J. \& Karplus, M. (1985) Proc. Natl. Acad. Sci. US 82, 1697

15. Snow, M.E. (1986) PhD Dissertation. The Johns Hopkins University School of Medicine.

16. Snow, M.E. \& Amzel, L.M. (1986) Proteins Struct. Funct. Genet. 1, 267
17. Moult, J. \& James, M.N.G. (1986) Proteins Struct. Funct. Genet. 1, 146

18. Bruccoleri, R.E. \& Karplus, M. (1987) Biopolymers 26, 137

19. Summers, N.L. \& Karplus, M. (1989) J. Mol. Biol. 209, 785

Address

Mark E. Snow

Scientific Computation Group

University of Michigan

535 West William Street

Ann Arbor, Michigan 48103-4943

USA 